\title{
Association of corrected QT interval with long-term mortality in patients with syncope
}

\author{
Nivas Balasubramaniyam¹, Chandrasekar Palaniswamy², Wilbert S. Aronow ${ }^{2}$, Sahil Khera ${ }^{1}$, \\ Gokulakrishnan Balasubramanian², Prakash Harikrishnan ${ }^{1}$, Jay V. Doshi ${ }^{2}$ Christopher Nabors ${ }^{1}$, \\ Stephen J. Peterson ${ }^{1}$, Sachin Sule ${ }^{1}$
}

\begin{abstract}
1Department of Medicine, Division of Internal Medicine, Westchester Medical Center/ New York Medical College, Valhalla, New York, USA

2Department of Medicine, Cardiology Division, New York Medical College at Westchester Medical Center, Valhalla, New York, USA

${ }^{3}$ Department of Medicine, Division of Internal Medicine, Rosalind Franklin University of Medicine and Science, North Chicago, Illinois, USA
\end{abstract}

Submitted: 7 November 2012

Accepted: 20 December 2012

Arch Med Sci 2013; 9, 6: 1049-1054

DOI: 10.5114/aoms.2013.39383

Copyright (c) 2013 Termedia \& Banach

\author{
Corresponding author: \\ Wilbert S. Aronow MD, FACC, \\ FAHA \\ Cardiology Division \\ New York Medical College \\ Macy Pavilion, Room 138 \\ Valhalla, NY 10595 USA \\ Phone: (914) 4935311 \\ Fax: (914) 2356274 \\ E-mail: wsaronow@aol.com
}

\begin{abstract}
Introduction: The electrocardiographic parameters QRS duration, QRS-T angle and QTc can predict mortality in patients with cardiovascular disease. The prgnostic value of these parameters in hospitalized patients with syncope needs investigation.

Material and methods: We retrospectively studied 590 consecutive patients hospitalized with syncope. After excluding patients with baseline abnormal rhythm, QT- prolonging medications, and missing data, 459 patients were analyzed. Baseline demographic characteristics, co-morbidities, medication use, San Francisco Syncope Rule (SFSR) and Osservatorio Epidemiologico sulla Sincope nel Lazio (OESIL) score and data on mortality were collected. The categorical variables and continuous variables of the 2 groups of patients with prolonged QTc and normal QTc interval were analyzed by Fischer's exact test and Mann-Whitney Test. A stepwise Cox regression model was used for time to death analysis. Results: Of 459 patients, prolonged QTc interval was observed in 122 (27\%). Mean follow-up was 41 months. Patients with prolonged QTc interval had higher prevalence of cardiovascular disease, OESIL score, high risk SFSR, hypertension, dyslipidemia, coronary artery disease, congestive heart failure, and increased mortality. Stepwise Cox regression analysis showed that significant independent prognostic factors for time to death were prolonged QTc interval $(p=0.005)$, age $(p=0.001)$, diabetes mellitus $(p=0.001)$ and history of malignancy $(p=0.006)$. QRS duration and QRS-T angle were not independent predictors of mortality.

Conclusions: A prolonged QTc interval is an independent predictor of long-term mortality in hospitalized patients with syncope.
\end{abstract}

Key words: QTc interval, syncope, mortality.

\section{Introduction}

Electrocardiographic parameters have been associated with increased mortality in patients with cardiovascular disease. These may include: QRS duration [1-3], QRS-T angle [4, 5], corrected QT interval [6], QTc dispersion $[7,8]$, and heart rate variability [9]. The QT interval represents the time from the onset of ventricular depolarization to the end of repolarization. 
This on the surface electrocardiogram (ECG) is measured from the onset of $\mathrm{Q}$ wave to the end of the T wave [10]. QT interval varies with heart rate and needs to be corrected for the same. Among the several methods available to correct the heart rate, Bazzett's formula is commonly used. Patients with congenitally long QTc syndrome have increased incidence of cardiac arrest or sudden cardiac death which may be the presenting symptom in as much as $13 \%$ of untreated patients [11]. Although prolonged QTc increases mortality in certain subgroups, its clinical significance and value in predicting total and cardiovascular mortality in the general population remains unclear [12]. QTc interval has been associated with increased mortality in patients with coronary heart disease $[13,14]$, diabetes mellitus [15], and congestive heart failure [16]. The association of QTc interval and mortality in patients hospitalized with syncope has not been adequately studied [6]. In this study, we report the association of various electrocardiographic parameters (QTc prolongation, QRS duration and QRS-T angle) with long-term mortality among patients presenting with syncope.

\section{Material and methods}

We retrospectively studied 590 consecutive patients hospitalized with syncope from January, 2007 to October, 2010 after obtaining Institutional Review Board approval. A total of 94 patients with paced rhythm (16), bundle branch block (51), atrial fibrillation (23) and atrial flutter (4) were excluded from the study as this would lead to inaccuracies in measurement of electrocardiographic parameters. Due to missing data (in 15 patients) and patients currently taking QT-prolonging medications (22), 37 more patients were excluded. A total of 459 patients were included in the final analysis.

Table I. Demographics and outcomes according to QTc interval

\begin{tabular}{|c|c|c|c|}
\hline Variables & $\begin{array}{c}\text { Normal QTC interval } \\
\leq 450(M), \leq 460(\mathrm{~F}) \\
(n=337)\end{array}$ & $\begin{array}{c}\text { Prolonged QTc interval } \\
\text { QTC }>450 \mathrm{~ms}(\mathrm{M}),>460 \mathrm{~ms}(\mathrm{~F}) \\
(n=122)\end{array}$ & Value of $p$ \\
\hline Men, $n(\%)$ & $178(53)$ & $72(59)$ & 0.25 \\
\hline Age, mean \pm SD [years] & $65.6 \pm 16.8$ & $65.3 \pm 17.1$ & 0.98 \\
\hline Caucasian race, $n$ (\%) & $245(73)$ & $85(70)$ & 0.56 \\
\hline Prior syncope, $n$ (\%) & $80(24)$ & $28(23)$ & 0.99 \\
\hline Prior cardiovascular disease, $n(\%)$ & $109(32)$ & $54(44)$ & 0.02 \\
\hline OESIL! Score & & & $<0.001$ \\
\hline High risk SFSR\$ score, $n(\%)$ & $191(57)$ & $96(79)$ & $<0.001$ \\
\hline Diabetes mellitus, $n$ (\%) & $74(22)$ & $36(30)$ & 0.1 \\
\hline Hypertension, $n(\%)$ & $219(65)$ & $101(83)$ & $<0.001$ \\
\hline Dyslipidemia, $n$ (\%) & $153(45)$ & $70(57)$ & 0.02 \\
\hline Coronary artery disease, $n(\%)$ & $73(22)$ & $44(36)$ & 0.002 \\
\hline Congestive heart failure, $n(\%)$ & $6(2)$ & $14(11)$ & $<0.001$ \\
\hline $\begin{array}{l}\text { Cerebrovascular accident/transient } \\
\text { Ischemic attack, } n(\%)\end{array}$ & $28(8)$ & $17(14)$ & 0.08 \\
\hline History of malignancy, $n$ (\%) & $35(10)$ & $21(17)$ & 0.054 \\
\hline Smoking, $n(\%)$ & $67(20)$ & $30(25)$ & 0.3 \\
\hline Glomerular filtration rate $\left[\mathrm{ml} / \mathrm{min} / 1.73 \mathrm{~m}^{2}\right]$ & $72 \pm 28$ & $72 \pm 29$ & 0.56 \\
\hline QRS duration [ms] & $90 \pm 12$ & $109 \pm 31$ & $<0.001$ \\
\hline QRS T angle [degrees] & $47 \pm 41$ & $74 \pm 55$ & $<0.001$ \\
\hline Vasovagal syncope, $n(\%)$ & $70(21)$ & $26(21)$ & 0.89 \\
\hline Syncopal etiology undetermined, $n$ (\%) & $103(30)$ & $45(37)$ & 0.21 \\
\hline Volume depletion, $n$ (\%) & $32(9)$ & $18(15)$ & 0.12 \\
\hline Cardiac causes of syncope,$n(\%)$ & $83(25)$ & $15(12)$ & 0.004 \\
\hline Mortality, $n(\%)$ & $41(12)$ & $31(25)$ & 0.001 \\
\hline
\end{tabular}

${ }^{*}$ Atrial and ventricular arrhythmias, second-degree or third-degree atrioventricular block, acute coronary syndrome, aortic stenosis, and hypertrophic obstructive cardiomyopathy; M - men, F-women, ! Osservatorio Epidemiologico sulla Sincope nel Lazio score, \$San Francisco syncope rule 
Cases were defined as those with QTc prolongation while controls were those with normal QTc intervals (based upon corrected QTc interval more than $450 \mathrm{~ms}$ in males and $460 \mathrm{~ms}$ in females is considered prolonged) [17].

Baseline demographic characteristics and comorbidities are shown in Table I. Mean follow-up was $41 \pm 18$ months. The diagnostic work-up for syncope included blood pressure recordings with orthostatic changes, electrocardiograms, cardiac biomarkers, telemetry, Holter recordings, and 2-dimensional echocardiograms in all patients. Computed tomography or magnetic resonance imaging scans of the head, carotid ultrasonograms, stress testing, coronary angiograms, and electrophysiology studies were also performed in selected patients if considered appropriate by the treating physician. The various etiologies identified for syncope are summarized in Table II.

We calculated the risk prediction scores for syncope - Osservatorio Epidemiologico sulla Sincope nel Lazio (OESIL) risk score [18] and San Francisco syncope rule (SFSR) [19] in all our patients. The OESIL score is calculated by the presence of the following 4 characteristics which indicate poor outcome: age $>65$ years, history of cardiovascular disease, syncope without prodrome, and an abnormal ECG [18]. High risk SFSR score indicates presence of at-least one of the following risk factors: abnormal ECG, a complaint of dyspnea, a hematocrit less than $30 \%$, a systolic blood pressure less than $90 \mathrm{~mm} \mathrm{Hg}$, or a history of congestive heart failure [19]. Prior cardiovascular disease is defined as the presence of one of the following: any form of structural heart disease, prior or current evidence of congestive heart failure, peripheral arterial disease, stroke or transient ischaemic attack. Electrocardiogram (ECG) is considered abnormal by the presence of rhythm abnormalities, atrio-ventricular or intraventricular conduction delay, past myocardial infarction, or ST-T changes consistent with or related to myocardial ischemia. Data on mortality were collected online from vital statistics website using 3 patient identifiers (name, date of birth, and social security number).

All patients had ECGs recorded with a Philips Pagewriter Touch Interpretive ECG Machine and stored using the Phillips TraceMaster Vue ECG management system version C.01. The TraceMasterVue ECG management system is a computer system that allows viewing, manual editing, printing, and archiving of digitally stored ECG data. The QT interval that was calculated by the computerized software was manually validated individually by 2 of our authors who were blinded on the outcome data. For QT interval validation, each individual lead was magnified to fit a 15.5 inch high resolution screen. An electronic caliper calibrated
Table II. Etiology of syncope on work-up in 459 patients

\begin{tabular}{|lc|}
\hline Etiology of syncope & Results, $n$ (\%) \\
\hline Vasovagal & $96(21)$ \\
\hline Volume depletion & $50(11)$ \\
\hline Etiology undetermined & $148(32)$ \\
\hline Orthostatic hypotension & $15(3)$ \\
\hline Sick sinus syndrome & $16(3)$ \\
\hline $\begin{array}{l}\text { Advanced second-degree or } \\
\text { third-degree atrioventricular block }\end{array}$ & $11(2)$ \\
\hline Supraventricular tachycardia & $8(2)$ \\
\hline Ventricular tachycardia & $23(5)$ \\
\hline Acute coronary syndrome & $22(5)$ \\
\hline Aortic stenosis & $13(3)$ \\
\hline Intracranial causes & $9(2)$ \\
\hline Drug overdose & $8(2)$ \\
\hline Hypoglycemia & $6(1)$ \\
\hline Hypertrophic obstructive cardiomyopathy & $4(1)$ \\
\hline Carotid sinus hypersensitivity & $4(1)$ \\
\hline Other causes & $26(5)$ \\
\hline
\end{tabular}

to accurately measure to the nearest $0.1 \mathrm{~mm}$ was used to calculate the QT interval. QT interval was measured from the beginning of the $\mathrm{Q}$ wave to the visual return of the $T$ wave. If the end of the $T$ wave was interrupted by a $U$ wave, the nadir between the $T$ and $U$ wave was taken as end of the $T$ wave. QTc interval was calculated in two consecutive beats in all leads, and the mean was taken as QTc interval duration. The heart rate correction of QT interval (QTC) was calculated using the Bazett's formula. As per the American Heart Association Electrocardiography and Arrhythmias Committee, Council on Clinical Cardiology, the American College of Cardiology Foundation, and the Heart Rhythm Society recommendation, a QTc interval more than $450 \mathrm{~ms}$ in men and $460 \mathrm{~ms}$ in women is considered prolonged [17].

\section{Statistical analysis}

All of the data were collected in Microsoft excel version 2010 (Redmond, Washington). The categorical variables and continuous variables were analyzed by Fischer's exact test and the Mann-Whitney Test. A stepwise Cox regression model was created to predict the time to death. The Kaplan-Meier curves were created to find out the survival rates of those with and without QTc prolongation. The log rank test (Mantel-Cox method) was used to compare these survival curves. All the data were analyzed using SPSS 20 version (Chicago, IL). A $p$ value of less than 0.05 is considered statistically significant. 


\section{Results}

Of the 459 patients, mean age $65 \pm 17$ years, included in the final analysis, 250 (54\%) were men. Of 459 patients, 108 (23\%) had a history of prior syncope, 110 (24\%) had diabetes mellitus, and $117(25 \%)$ had a prior diagnosis of coronary artery disease. Of these 459 patients, 122 (27\%) had a prolonged QTc interval. The demographics of the patients by QTc interval are shown in Table I. Patients with a prolonged QTc interval had a higher prevalence of cardiovascular disease, OESIL score, high risk SFSR, hypertension, dyslipidemia, coronary artery disease, congestive heart failure, QRS duration, QRS- T angle, and increased mortality.

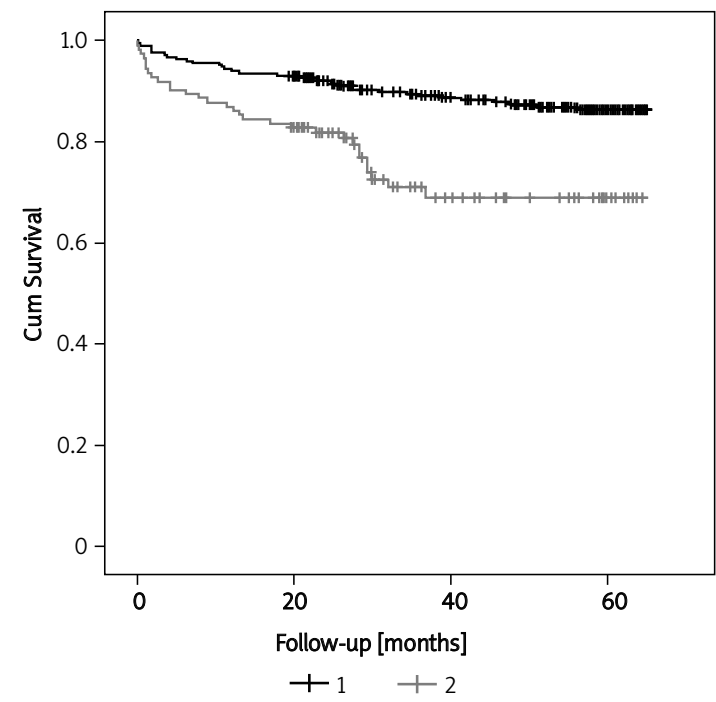

Figure 1. Kaplan-Meir survival curves for those with and without prolonged QTc interval $\left(p<0.001^{*}\right)$ ${ }^{1} \mathrm{QTC}>450(\mathrm{M}),>460(\mathrm{~F}),{ }^{2} \mathrm{QTC} \leq 450(\mathrm{M}), \leq 460(\mathrm{~F}),{ }^{*} p$ value by log rank test
The etiologies of syncope in our study population are shown in Table II. Idiopathic (32\%), vasovagal (21\%), and volume depletion (11\%) were the common etiologies in our study population in descending order. During a mean follow-up of 41 \pm 18 months, 72 patients (16\%) died.

Table III shows the results of multivariate analysis to determine the independent predictors of mortality in patients hospitalized with syncope. All characteristics in Table I that were found significant on univariate analysis along with age and sex were included in stepwise Cox regression analysis to determine predictors of time to death. There was no significant difference in survival between men and women admitted with syncope. QRS duration was not significant in the univariate analysis to predict mortality $(p=0.11)$, and hence not included in stepwise Cox regression analysis. Prolonged QTC (> $450 \mathrm{~ms}$ in men and $>460 \mathrm{~ms}$ in women) was an independent predictor of mortality $(p=0.005)$ along with age $(p=0.001)$, diabetes mellitus $(p=0.001)$, and history of malignancy $(p=0.006)$. QRS duration and QRS-T angle were not significant independent predictors of mortality. Figure 1 shows the Kaplan-Meier survival curves for those with and without a prolonged QTc interval.

In addition, we performed a subgroup analysis looking at the predictors of mortality in patents without structural heart disease. Multivariate analysis by Cox proportional hazards showed that the only significant independent predictor of mortality in the 378 patients without structural heart disease was a prolonged QTc interval (odds ratio $=2.427$; 95\% Cl: 1.323-4.452; $p=0.004)$.

We compared the sensitivity and specificity of the OESIL and SFSR scores to determine long-term mortality ( $41 \pm 18$ months) with the QTc interval alone and in combination. Scores of $\geq 1$ and $\geq 2$ are

Table III. Cox proportional hazards ratios to calculate independent prognostic factors for long- term mortality

\begin{tabular}{|c|c|c|c|c|}
\hline \multirow[t]{2}{*}{ Variable } & \multirow[t]{2}{*}{ Value of $p$} & \multirow[t]{2}{*}{ Hazards ratio } & \multicolumn{2}{|c|}{$95 \% \mathrm{Cl}$ for $\operatorname{Exp}(\mathrm{B})$} \\
\hline & & & Lower & Upper \\
\hline Age & 0.001 & 1.041 & 1.017 & 1.065 \\
\hline Sex & 0.198 & 0.716 & 0.430 & 1.191 \\
\hline OESIL score! & 0.213 & & & \\
\hline Prior cardiovascular disease & 0.238 & 1.557 & 0.747 & 3.245 \\
\hline SFSR high risk $\$$ & 0.107 & 1.751 & 0.885 & 3.464 \\
\hline Diabetes mellitus & 0.001 & 2.271 & 1.369 & 3.766 \\
\hline Prior cerebrovascular accident or transient ischemic attack & 0.288 & 1.440 & 0.735 & 2.822 \\
\hline QRS T angle & 0.530 & 1.002 & 0.996 & 1.007 \\
\hline History of malignancy & 0.006 & 2.350 & 1.284 & 4.302 \\
\hline QTc $>460 \mathrm{~ms}$ in women and $>450 \mathrm{~ms}$ in men & 0.005 & 2.115 & 1.259 & 3.552 \\
\hline Glomerular filtration rate & 0.311 & 0.995 & 0.985 & 1.005 \\
\hline
\end{tabular}

! Osservatorio Epidemiologico sulla Sincope nel Lazio score, \$San Francisco syncope rule 
considered high-risk SFSR and OESIL, respectively. As a single test, high-risk SFSR has the highest sensitivity (0.54) compared to high-risk OESIL (0.33) and QTc prolongation (0.25). QTc prolongation and high-risk OESIL score have a comparable specificity (0.73) which is better than a high-risk SFSR score (0.54). When combining these tests, QTc prolongation or high-risk SFSR score or high-risk OESIL score has the highest sensitivity (0.75). QTc prolongation plus high-risk OESIL has the highest specificity (0.96).

\section{Discussion}

In this retrospective study, we found that a prolonged QTc interval independently predicted mortality in patients admitted to the hospital with the diagnosis of syncope. The process of repolarization in the ventricular myocardial cells is not entirely homogenous. If this heterogeneity that normally exists is increased, it results in prolongation of the QT interval. This increased heterogeneity can predispose to the development of malignant ventricular arrhythmias, including torsades de pointes [14]. In patients with underlying cardiac disease, ventricular repolarization heterogeneity is increased resulting in QTc interval prolongation [15]. Although prolonged QTc interval is suggested to come from sympatho-vagal balance, the primary determinant of QTc interval is left ventricular systolic performance [16]. Also, men with prolonged QTc interval are at a higher risk of coronary artery disease and related mortality independent of other risk factors [6]. Hence, prolonged QTc interval is a useful indicator that could help unravel underlying cardiac disease, which could predict long-term mortality. Prolonged QTc has also been shown to increase mortality in patients with diabetes mellitus [15] and congestive heart failure $[9,15]$.

Although corrected QT interval has been shown to predict mortality in specific subgroup of patients as mentioned above, its importance in the general population remains unclear. In a review of 7 prospective cohort studies looking at prolonged QTC and mortality in the general population [12], one study reported no association between prolonged QTC interval and mortality. The other six studies reported inconsistent associations overall and across subgroups. The only consistent finding was in the subgroup of patients with prior cardiovascular disease. The relative risks of prolonged QTc interval ranged from 1.1 to 3.8 for total mortality, 1.2 to 8.0 for cardiovascular mortality, and 1.0 to 2.1 for sudden death in patients with prior cardiovascular disease. The authors concluded that there was inconsistent evidence for association of QTc with total mortality, cardiovascular mortality, and sudden death, except perhaps in patients with prior cardiovascular disease [12].
In a retrospective study of 348 patients presenting to the emergency department with syncope, prolonged QTc interval was defined as a QTC interval $\geq 440 \mathrm{~ms}$ [6]. In this study, patients with a prolonged QTc interval had significantly higher mortality when compared to those with a normal QTc interval ( $22 \%$ vs. $11 \%$; $p=0.004)$. After adjustment for covariates in the Cox regression model, age $\geq 65$ years (HR 7.9; 95\% Cl: $1.9-32.9 ; p=0.004)$ and QTc interval $\geq 500 \mathrm{~ms}$ (HR 3.5; $95 \% \mathrm{Cl}: 1.56-$ 8.12; $p=0.002$ ) were predictors of increased mortality among patients with syncope. A QTc interval $\geq 440$ ms was not independently associated with increased mortality. The causes of syncope were not identified in that study population [6].

The SFSR and OESIL are commonly used risk prediction scores to evaluate short-term outcomes in patients with syncope. The SFSR score is the only risk predictor that has been validated in various settings. This simple, 5-step clinical decision score is intended to identify patients at risk of short-term serious outcomes $[19,20]$. In a recent meta-analysis, a pooled estimate of sensitivity of the San Francisco Syncope Rule was 0.87 (95\% Cl: 0.79-0.93). The pooled estimate of specificity was $0.52(95 \% \mathrm{Cl}$ : 0.43-0.62) [21].

Colivicchi et al. [18] derived the OESIL score based on an arithmetic sum of 4 important predictors of mortality at 1 year by multivariate analysis. These investigators identified a significant increase in mortality as the OESIL score increased, both in the derivation and validation cohort. In subsequent studies, a high-risk OESIL score had a sensitivity of $90 \%$ and a specificity of $76 \%$ for shortterm mortality [22].

The present study is the first study showing the sensitivity and specificity of the SFSR score, the OESIL score, and a prolonged QTc interval alone and in combination in predicting long-term mortality in patients with syncope. The SFSR score has the highest sensitivity, and the OESIL score and prolonged QTc have the highest specificity in predicting longterm mortality when used alone. When combining these tests, QTc prolongation or high-risk SFSR score or high-risk OESIL score has the highest sensitivity (0.75). QTc prolongation plus high-risk OESIL has the highest specificity (0.96).

Diabetes mellitus was a significant independent predictor of mortality ( $p=0.001)$ in our patients with syncope. In a recent study of 22,189 patients with 23,951 emergency department visits for syncope, diabetes ( $p=0.002)$ was identified as a significant independent predictor of 30-day mortality [23]. Our study identified syncope as a predictor of long-tem mortality.

The study has the limitations of being a retrospective study with lack of data regarding the cause of death. Although every effort was made to eval- 
uate differences in baseline variables that might have contributed to differences in mortality, there may have been other variables not accounted for that could have influenced outcome in this study. The present study was a single center study, and thus the results reflect the demographics and standard of care representative of a single region of the United States.

This study confirms the clinical utility of QTC interval as a risk prediction tool for long-term mortality in patients presenting with syncope due to various etiologies. We conclude that in patients hospitalized with the diagnosis of syncope, a QTc interval more than $450 \mathrm{~ms}$ in men and $460 \mathrm{~ms}$ in women is an independent predictor of long- term mortality along with age, history of malignancy, and diabetes mellitus.

\section{References}

1. Kurl S, Makikallio TH, Rautaharju P, et al. Duration of QRS complex in resting electrocardiogram is a predictor of sudden cardiac death in men. Circulation 2012; 125: 2588-94.

2. Lai HM, Aronow WS, Rachdev A, et al. Incidence of mortality in 1,040 patients with coronary heart disease or hypertensive heart disease with normal and abnormal left ventricular ejection fraction and with normal and abnormal QRS duration. Arch Med Sci 2008; 4: 140-2.

3. Kannam H, Aronow WS, Chilappa K, et al. Association of the QRS duration on the resting electrocardiogram with the severity of coronary artery disease in 2,196 patients undergoing coronary angiography for suspected coronary artery disease. Arch Med Sci 2009; 5: 163-5.

4. Whang W, Shimbo D, Levitan EB, et al. Relations between $\mathrm{QRS} / \mathrm{T}$ angle, cardiac risk factors, and mortality in the third national health and nutrition examination survey (NHANES III). Am J Cardiol 2012; 109: 981-7.

5. Lown MT, Munyombwe T, Harrison W, et al. Association of frontal QRS-T angle: age risk score on admission electrocardiogram with mortality in patients admitted with an acute coronary syndrome. Am J Cardiol 2012; 109: 307-13.

6. Aggarwal A, Sherazi S, Levitan B, et al. Corrected QT interval as a predictor of mortality in elderly patients with syncope. Cardiol J 2011; 18: 395-400.

7. Giunti S, Gruden G, Fornengo P, et al. Increased QT interval dispersion predicts 15 -year cardiovascular mortality in type 2 diabetic subjects: the population-based Casale Monferrato study. Diabetes Care 2012; 35: 581-3.

8. Okin PM, Devereux RB, Howard BV, et al. Assessment of QT interval and QT dispersion for prediction of all-cause and cardiovascular mortality in american indians: the Strong Heart study. Circulation 2000; 101: 61-6.

9. Tsuji H, Venditti FJ Jr, Manders ES, et al. Reduced heart rate variability and mortality risk in an elderly cohort: the Framingham Heart study. Circulation 1994; 90: 878-83.

10. Moss AJ. The QT interval and torsade de pointes. Drug Saf 1999; 21 (Suppl. 1): 5-10.

11. Priori SG, Schwartz PJ, Napolitano C, et al. Risk stratification in the long-QT syndrome. N Engl J Med 2003; 348: 1866-74.

12. Montanez A, Ruskin JN, Hebert PR, et al. Prolonged QTC interval and risks of total and cardiovascular mortality and sudden death in the general population: a review and qualitative overview of the prospective cohort studies. Arch Intern Med 2004; 164: 943-8.

13. Dekker JM, Schouten EG, Klootwijk P, et al. Association between $\mathrm{QT}$ interval and coronary heart disease in middleaged and elderly men: the Zutphen study. Circulation 1994; 90: 779-85.

14. Goldberg RJ, Bengtson J, Chen ZY, et al. Duration of the QT interval and total and cardiovascular mortality in healthy persons (the Framingham Heart study experience). Am J Cardiol 1991; 67: 55-8.

15. Rossing P, Breum L, Major-Pedersen A, et al. Prolonged QTc interval predicts mortality in patients with type 1 diabetes mellitus. Diabet Med 2001; 18: 199-205.

16. Vrtovec B, Delgado R, Zewail A, et al. Prolonged QTC interval and high $\mathrm{B}$-type natriuretic peptide levels together predict mortality in patients with advanced heart failure. Circulation 2003; 107: 1764-9.

17. Rautaharju PM, Surawicz B, Gettes LS, et al. AHA/ ACCF/ HRS recommendations for the standardization and interpretation of the electrocardiogram: Part IV: The ST segment, $\mathrm{T}$ and $\mathrm{U}$ waves, and the QT interval: a scientific statement from the American Heart Association electrocardiography and arrhythmias committee, Council on Clinical Cardiology; the American College of Cardiology Foundation; and the Heart Rhythm Society endorsed by the International Society for Computerized Electrocardiology. J Am Coll Cardiol 2009; 53: 982-91.

18. Colivicchi F, Ammirati F, Melina D, et al. Development and prospective validation of a risk stratification system for patients with syncope in the emergency department: the OESIL risk score. Eur Heart J 2003; 24: 811-9.

19. Quinn JV, Stiell IG, McDermott DA, et al. Derivation of the San Francisco syncope rule to predict patients with shortterm serious outcomes. Ann Emerg Med 2004; 43: 224-32.

20. Quinn J, McDermott D. ECG criteria of the San Francisco Syncope Rule. Ann Emerg Med 2011; 57: 72-3.

21. Saccilotto RT, Nickel CH, Bucher HC, et al. San Francisco Syncope Rule to predict short-term serious outcomes: a systematic review. CMAJ 2011; 183: E1116-26.

22. Kayayurt K, Akoglu H, Limon O, et al. Comparison of existing syncope rules and newly proposed anatolian syncope rule to predict short-term serious outcomes after syncope in the Turkish population. Int J Emerg Med 2012; 5: 17.

23. Derose SF, Gabayan GZ, Chiu VY, et al. Patterns and preexisting risk factors of 30-day mortality after a primary discharge diagnosis of syncope or near syncope. Acad Emerg Med 2012; 19: 488-96. 